\title{
MODULAR INDEPENDENT METERING SYSTEM FOR MOBILE APPLICATIONS PROVIDING SMOOTH MODE TRANSITION
}

\author{
Jan Lübbert ${ }^{1 *}$, Jürgen Weber ${ }^{1}$, Christian Stauch $^{2}$, Peter Bruck ${ }^{2}$ \\ Institute of Mechatronic Engineering, Technische Universität Dresden, Helmholtzstraße 7a, D-01069 Dresden, \\ Germany \\ HYDAC Fluidtechnik GmbH, Justus-von-Liebig-Straße, D-66280 Sulzbach/Saar, Germany \\ * Corresponding author: Tel.: +49 351463 33701; E-mail address: Jan_Frederik.Luebbert@tu-dresden.de
}

\begin{abstract}
Independent metering valve systems open up for more flexibility because of the ability to control meter in and meter out flow individually, thus enabling many possibilities to adapt the actuator's behaviour to the user's needs without altering any hardware. Furthermore, with alternative flow modes significant energy savings are possible. In many applications like excavators smooth switching between flow modes is required, a demand the market does not provide a satisfying solution for yet. Here, an approach using a short circuit path for smooth switching has been developed. The control algorithm hands over the volume flow from one path to another continuously depending on the current actuator load. Due to the flexible software structure the developed control algorithm can be applied to a very broad variety of independent metering valve layouts. Simulation results are promising and currently the solution is undergoing test rig evaluation.
\end{abstract}

Keywords: independent metering, mobile machines, energy efficiency, control strategy

\section{INTRODUCTION}

Hydraulic systems for actuating human controlled mobile machines have undergone a long history regarding development and optimisation. The state of the art changed from constant flow open centre systems, which emerged in the 1950s, over negative (NFC) and positive flow control systems (PFC) to load sensing and flow sharing [1]. Nowadays, after several decades of technological progress, these conventional systems satisfy most requirements regarding controllability, but they significantly lack energy efficiency - a topic becoming more and more important. One reason for the low of energy efficiency are unnecessary throttling losses at meter-out orifices of coupled-meteringvalves designed for control of altering, especially overrunning loads [2].

The common closed-centre load sensing (CCLS) and open-centre systems (OC) behave very different and are therefore more or less suited for different tasks. For example, when grabbing a pipe with an excavator bucket or accelerating a long and heavy boom, the good force control properties of OC-systems are desirable, but for finishing a surface it might be more desirable to have the good velocity control of an LS-flowsharing system. These different kinds of principal behaviour cannot be changed in a conventional system. So the customer must accept a trade-off regarding his type of hydraulic system and the intended application of the machine. On the other hand side OEMs need to equip their machines with different valve systems to meet the customer expectations in different countries. Furthermore they have to put a lot of effort into the design and tuning of complex valve spools. Another aspect becoming more and more important is automation. Coping with the cross interactions and load dependency of conventional systems to perform an accurate movement under varying load autonomously is a challenging task.

Independent Metering (IM) is the ideal technology to overcome the aforementioned limitations of common valve systems in mobile machines: Decoupling of inlet and outlet into separate valves eliminates the need for complex and application-specific spool design - standard valves can be applied to any task with comparable flow requirements. With adaption of the opening ratio between inlet and outlet throttle by means of IM controllability and energy efficiency can be 
improved at the same time. The energetic savings are obtained by prevention of unnecessary throttling of the outlet flow and by utilisation of regenerative flow circuits. Also automation and productivity can be facilitated by IM, since this technology enables complete load compensation.

\section{MODE SHIFTING PROBLEM AND STATE OF THE ART}

The major part of the energetic benefits of IM lies in the alternative flow modes. The feasibility and efficiency of these modes depends on the load. In case of an excavator, loads are not predictable in advance, so it must be possible to change the flow mode quickly during an ongoing movement without irritating the operator. To make IM suitable and attractive for mobile machines especially excavators - the challenging task of mode switching must be solved.

The easiest way to cope with mode shifting is to prevent mode shifting during the movement. This can be accomplished by predefining suitable flow modes for typical movements and loads [3]. The mayor drawback of this approach is the fact, that loads change during the movement and without shifting into the optimal mode energy efficiency decreases, for example when a high load must be pushed in high pressure regeneration, causing an unnecessarily high pump pressure. This would explain the relatively low energetic saving of around $10 \%$ in the cited publication. The mentioned kind of situations can also lead to functional problems, for example slowdown or stop of a movement.

There are two possible ways to shift between modes; discrete or continuous:

Discrete shifting implies an instant step from one mode to another. Pressure compensators can help to supress the impact of sudden changes in the pressure drops over the affected metering orifices. The flow path must by no means be disrupted during the mode shift, a demand that most currently available IM systems (Eaton CMA [4], Danfoss PVX [5], IM valve by Wessel Hydraulik [6]) do not fulfil. A discrete mode shift implies a sudden change in the pump flow demand on which the pump cannot react immediately because of its limited dynamics. The results are disturbances in the supply line which can negatively affect other consumers.

So the preferable way to avoid the aforementioned problems is continuous mode switching within a certain time or load window [7]. A market solution capable of continuous mode switching is INCOVA by Husco [8], but its major drawbacks are the missing compensation of supply disturbances and its need for a pressurised tank line for energy-neutral lowering. This would be a constant source of energy losses.

A solution to enable energy-neutral lowering without pressurizing the tank line is the shortcircuit-valve (SC). Such an IM valve system is not available on the market yet, even though it would reduce the number of proportional valves needed for continuous mode switching from four to three.

\section{SYSTEM ARCHITECTURE AND CONTROL ALGORITHMS}

This section starts with the general IM valve structure, followed by the software architecture. Afterwards the key functionalities velocity and pressure control as well as mode shifting will be explained.

\subsection{General independent metering valve system}

In [9] a general IM system has been proposed which consists of five flow paths (Figure 1), marked with arrows in the figure, together with additional paths which will be referred to later. These five flow paths are PK from pump (P) to piston side node (K), KT from piston side node to tank, PS from pump to rod side node (S), ST from rod side node to tank and KS from piston side node to rod side node. In addition to the work in [9] the two paths $\mathrm{K}$ and $\mathrm{S}$ for safety shut-off or counterbalance valves at the cylinder ports have been added. Most IM system can be classified as special cases of this system comprising all or a subset of these flow paths.

So far, according to Figure 1, the general IM system consists of up to seven 2/2-valves. Controlling each valve as a separate element would exclude any 3/3- or 4/3-valves. Therefore the circuit is divided into subcircuits (grey in the figure with bold names and italic ports): PATK with its ports $\mathrm{P}, \mathrm{A}$ and $\mathrm{T}$ connects the piston side node $\mathrm{K}$ with pressure or tank. PATS does the same with the node S. KS is the short circuit path and the safety valves are also separate subcircuits.

The IM control software does not refer to the individual valves as actor elements but only to encapsulated control code of these subcircuits. 
Hence, these subcircuits can be implemented with any hardware. The interfaces are the flows $Q_{i}$ in Figure 1 and the pressures at the ports of the subcircuits. This architecture makes the control software on axis level independent from the valve circuit layout. A change in the valve circuit layout affects only the control software of the corresponding subcircuit rather than the complete control software of the axis.

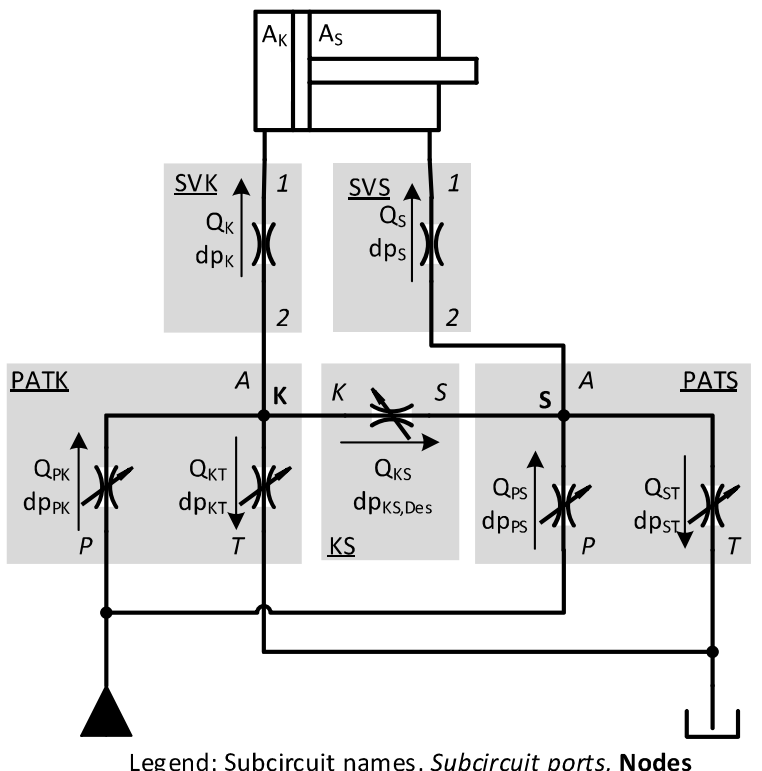

Figure 1: General independent metering valve system

\subsection{Software Architecture}

The architecture of the developed control software is shown in Figure 2. For the sake of simplicity as an example only the path from the operator input to the valve PK (see Figure 1) is displayed. The complete architecture comprises all axes, all subcircuits and all valves.

The top end of the control chain is the machine controller, which receives the desired velocities $v_{\text {Des }}$ from the joysticks. The machine controller hands over the velocity commands $v_{\text {Des }}$ to the individual axis controllers, in this case axis 1 . The axis controller measures the cylinder load using pressure sensors and selects the flow mode. From the mode and velocity command it calculates the required pump flow $Q_{1, R e q}$ and pressure $p_{1, R e q}$ and provides these values to the machine controller. The machine controller then determines the overall pump flow $Q_{0, R e q}$ and pressure $p_{0, R e q}$ and sends these to the pump controller.

The axis controller also selects and executes the velocity and pressure control scheme. This algorithm is independent from the flow mode. From the pressure and velocity controller and the current flow mode the axis controller derives the pressure, flow or conductance commands for the subcircuits (in Figure $2 p_{K, \text { Des }}$ and $Q_{P K, \text { Des }}$ for PATK) and hands these values over to the respective subcircuit controller, in the example PATK.

Gathering possibly required information (depending on the functionality requested by the axis controller) from assigned sensors - in the example the pump pressure $p_{P, \text { Meas }}$ the subcircuit controller calculates the set values for its valves. These are conductances $K$ for throttling elements, pressures $p$ for pressure control valves or volume flows $Q$ for flow control valves.

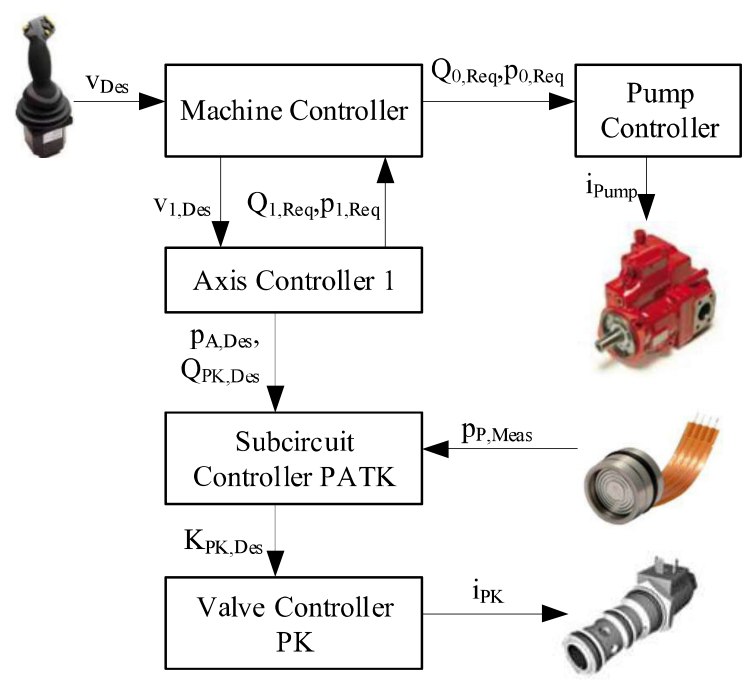

Figure 2: General software architecture

The corresponding valve controller then derives the required spool current $i$ from the set value $K$, $p$ or $Q$. The valve controllers can comprise calibration or learning algorithms.

With the proposed software architecture all subcircuits and their valves are completely interchangeable without affecting the axis controller as long as the overall valve circuit can be classified as a special case of the general valve layout shown in Figure 1.

\subsection{Velocity and Pressure Control}

Independent metering allows for the control of the two variables pressure level and volume flow of a hydraulic consumer independently. The IM operation strategy defines the desired inlet chamber pressure $p_{\text {in,Des }}$ and velocity $v_{\text {Des }}$ of the actuator, while the IM control strategy calculates the required set values for the flow paths to 
actually obtain the desired pressure level and velocity.

IM Operation Strategy. The IM operation strategy defines the desired inlet chamber pressure of the consumer depending on its current load, measured with pressure sensors. The goal of the operation strategy is to make the load range, within which energy saving regenerative flow modes are feasible, as large as possible.

In the case of extension the pressure level is set as high as possible without raising the pump pressure. This means that the highest loaded consumer is always running on the lowest possible pressure level (outlet pressure as low as possible, inlet pressure depending on the load) and the lower loaded consumers' pressure levels are artificially raised to nearly pump pressure. This raises the outlet pressure to a higher level than the inlet pressure in a wide load range and thus enables high pressure flow regeneration over the short circuit valve.

Retracting movements are carried out at the lowest possible outlet pressure in case of resistive loads and with a constant pressure drop between outlet and inlet for aiding loads. The latter leads to a relatively high pressure level in the cylinder and enables energy neutral low pressure regeneration over the $\mathrm{SC}$ valve in a wide load range and high pressure flow feedback into the pump line while lowering heavy loads.

IM Control Strategy. For controlling pressure and velocity in a hydraulic consumer with IM there exist different approaches. Since an
IM system is a multi-input-multi-output system (MIMO), corresponding MIMO control approaches like applied in [9] would suit best for the task in theory, but concerning mobile applications uncertain plant parameters and wide variations of these should be considered. For example the eigenfrequencies of an excavator boom change drastically depending on its position and payload. This parameter change cannot be taken into account by the control since the implement position is usually not measured. Therefore a robust SISO control approach has been chosen here: On one workport the velocity is controlled by means of flow control - either electronically or by means of a flow control valve, while on the other workport the pressure is controlled.

Figure 3 shows an example implementation of a SISO IM control algorithm: With the piston areas $A_{I n}$ and $A_{\text {Out }}$ the required volume flows $Q_{\text {In }}$ and $Q_{\text {out }}$ are calculated from the desired velocity $v_{\text {Des }}$. On the inlet side the chamber pressure is controlled. Therefore a pressure controller compares the measured inlet pressure $p_{\text {In,meas }}$ to the desired value $p_{\text {In,Des }}$ and calculates a volume flow $Q_{P C}$ which is added to $Q_{I n}$. With the sum flow $Q_{I n}+Q_{P C}$ and the measured pressure drop $\Delta p=p_{0}-p_{\text {In,Meas }}$ over the inlet valve its conductance $K_{I n}$ is determined. On the outlet side the velocity is controlled by means of an electronically pressure compensated volume flow: Assuming zero tank pressure the pressure drop over the outlet valve equals the

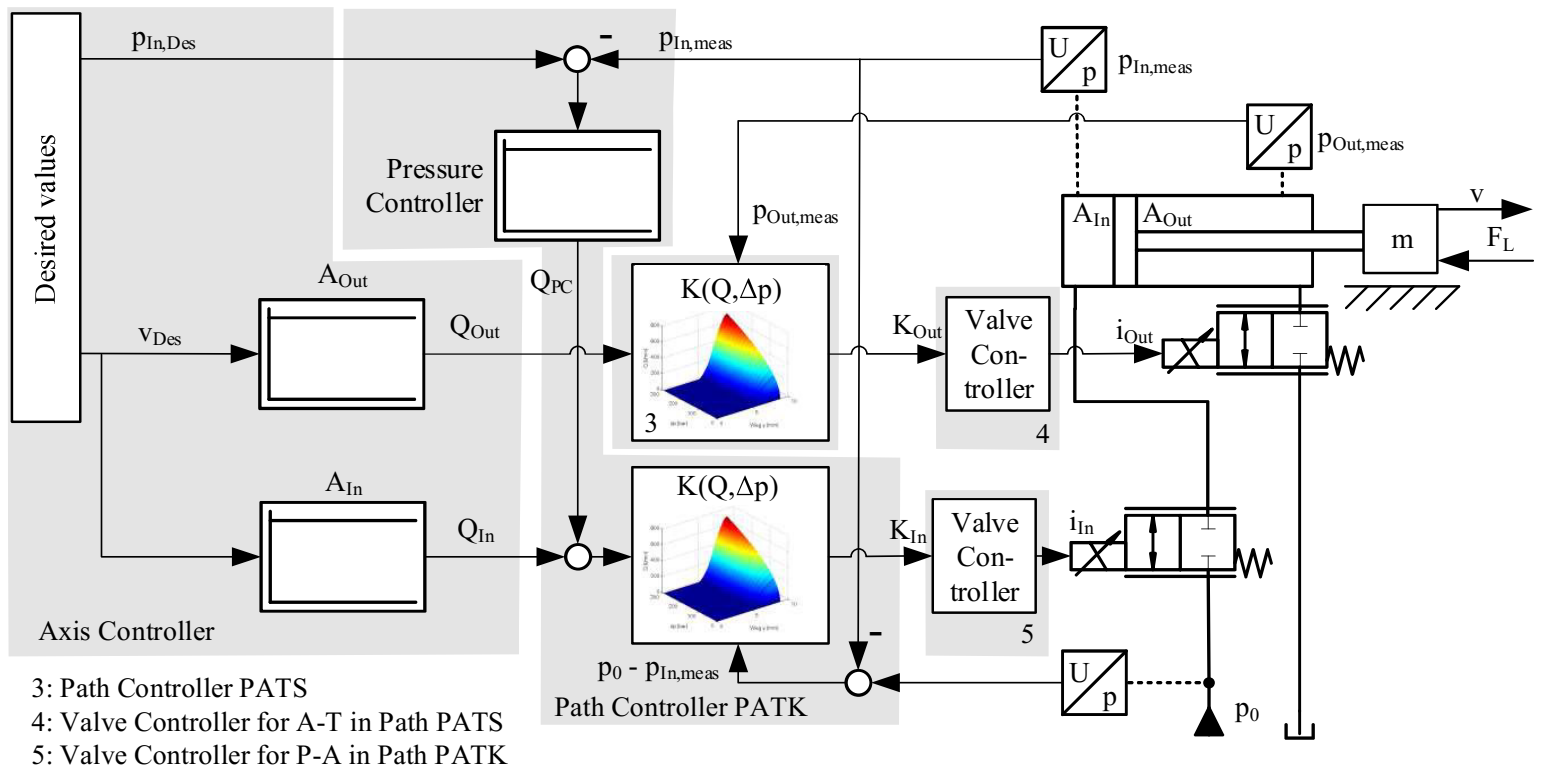

Figure 3: Example of SISO flow and pressure control 
outlet chamber pressure $p_{\text {Out,meas }}$. With this and $Q_{\text {out }}$ the outlet valve's conductance $K_{\text {Out }}$ is calculated.

Since this control scheme refers to the workports of the consumer it is independent from the flow mode - switching between flow modes only hands over the control task on the workport to another valve. Thus, disruptions of the pressure and flow control process, as encountered in [10], are circumvented.

The results of a linear MIMO coupling factor analysis in [2] showed that the velocity of resistive loads should be controlled on the inlet side and the pressure on the outlet side. With aiding loads it is advisable to swap these two functions to keep the dynamic cross-interactions between pressure and flow control minimal. To account for this, it is possible to add such a pressure and flow control swap functionality in the software if test rig results indicate the necessity to do so.

\subsection{Flow modes and mode shifting algorithm}

The principal approach to smooth mode shifting used here has been initially developed by Shenouda [7]. A smooth mode shift requires a continuous transition between two distinct flow modes, thus leading to a transition mode, called continuously variable mode (CVM). While Shenouda worked with a Wheatstone bridge valve circuit his approach has been adapted to a short circuit layout here. In the following the mode transition between normal extension NM+ and high pressure short circuit regeneration extension with node on piston side (hpREGscK + ), both special cases of their common CVM NMhpREGscK+, will be explained as an example (Figure 4):

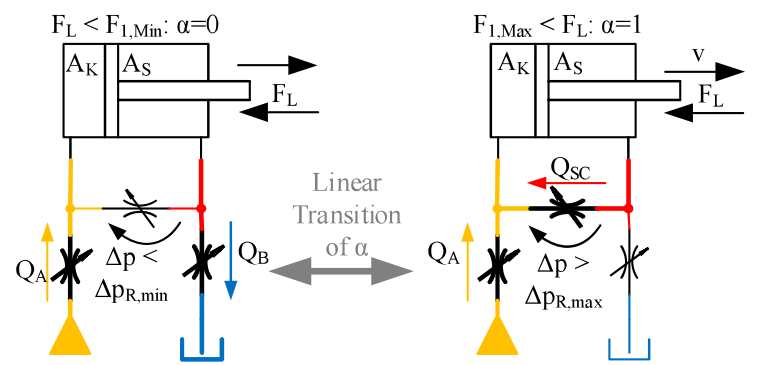

Figure 4: Flow modes during extension

The transition takes place in a load force window
$F_{1, \min }<F_{L}<F_{1, \max }$

where the force thresholds $F_{1, \min }$ and $F_{1, \max }$ are defined by minimum and maximum pressure drop over the short circuit path. Within this force window the mode shifting variable $\alpha$ transitions from 0 to 1 in a linear manner. With $\alpha$ between 0 and 1 three flow paths are active, since the outlet flow $v A_{S}$ is divided between tank line $\left(Q_{B}\right)$ and short circuit $\left(Q_{S C}\right)$ :

$Q_{A}=v\left(A_{K}-\alpha A_{S}\right)$

$Q_{B}=(1-\alpha) v A_{S}$

$Q_{S C}=\alpha v A_{S}$

During a continuous mode transition the outlet flow is "handed over" smoothly between tank and short circuit line.

To prevent unnecessary mode switching fluctuating load forces should be filtered before comparing them to the shift level.

\section{TAYLORING TO SPECIFIC APLICATION}

\subsection{Sample application case and energetic potential}

Sample application. As a sample application a medium sized excavator of $20 \mathrm{t}$ weight has been chosen. Its hydraulic system is characterized by a relief pressure of 350 bar and peak volume flows of $200 \mathrm{l} / \mathrm{min}$ per axis. In the field of mobile machines the excavator is the most demanding machine type regarding the requirements on motion controllability: During typical excavating and handling tasks several consumers are operated in parallel under varying loads and velocities. Therefore, to exploit the full energetic potential of IM, mode shifting during movements is crucial.

Energetic analysis. For the sake of cost and complexity reduction only the operation modes of significant energetic relevance shall be implemented. This reduces the number of required flow paths and valves and mode switching events. To identify these energetically attractive modes an energetic analysis according to [9] has been performed. Force and displacement profiles $x(t)$ and $v(t)$ serve as input for the analysis. The overall worktime of the excavator has been divided into realistic portions for different tasks. These tasks and their corresponding time shares are listed in Table 1. 
Table 1: Work tasks for excavator

\begin{tabular}{lcc}
\hline \multicolumn{1}{c}{ Work task } & $\begin{array}{c}\text { Time share } \\
{[\%]}\end{array}$ & Source of profile \\
\hline Power digging & 64 & Experiment \\
Pulled levelling & 10 & Synthetic \\
Pushed levelling & 10 & Synthetic \\
Slope grading & 9,5 & Synthetic \\
Utility handling & 6,5 & Synthetic \\
\hline
\end{tabular}

The power digging task has been carried out as an experiment with the real excavator in grown soil, while the other tasks have been defined as synthetic loads and motions of the tool centre point (TCP) at the bucket tip or bucket joint respectively. These motion and load profiles of the TCP have been transformed into the corresponding cylinder profiles be means of an analytical kinematic and kinetic model of the machine. As an example for this procedure the slope grading cycle is displayed in Figure 5. The solid lines represent the excavator implement's position at the beginning, while the dashed lines show the movement of the links' end points over the whole cycle. The resulting cylinder forces are shown black dashed and the strokes in solid purple.

The method in [9] identifies the energetically optimal combination of flow modes and the corresponding flows and pressures for the valves according to Figure $\mathbf{1}$ and for the pump for each time step individually, taking into account the given restrictions regarding pressure, volume flow and flow coefficient of the paths. Hence, the algorithm assumes ideal, infinitely fast switching. So far all theoretically possible modes listed in [9] are available and therefore the result is the highest theoretically possible energy saving.
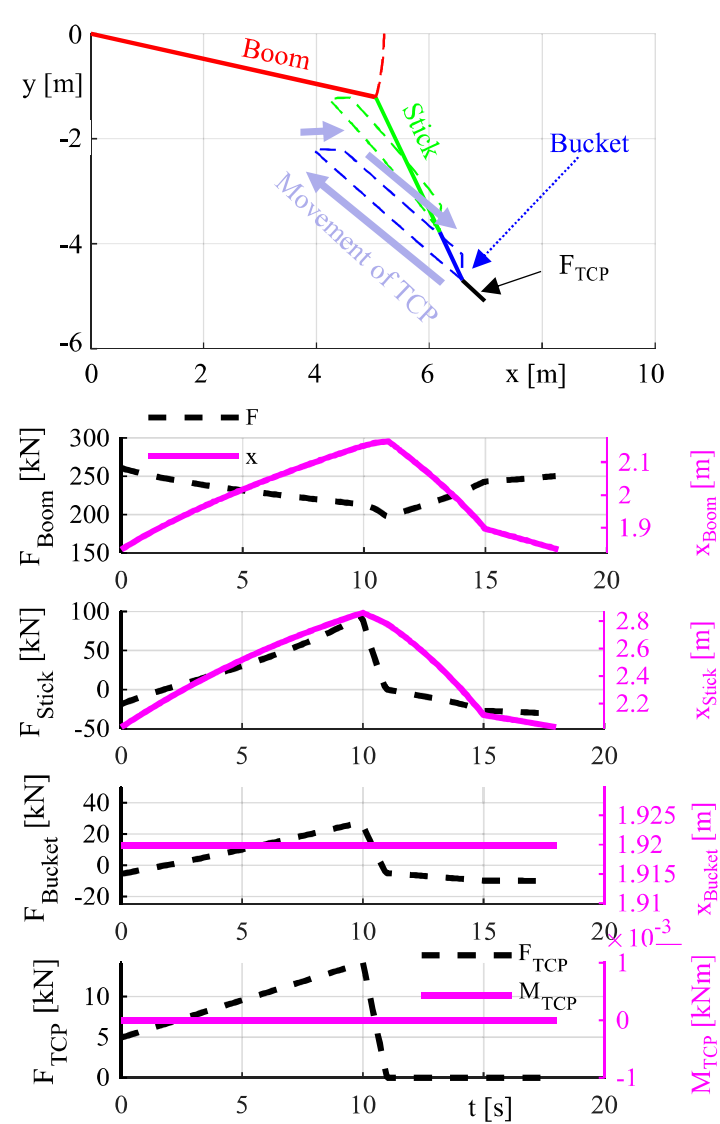

Figure 5 : Movement and cylinder loads for grading

To find an optimal combination of only a few flow modes to implement the method has been adapted: Each mode can be allowed or forbidden individually. The analysis is repeated many times automatically while systematically allowing or forbidding different modes. By doing so, an energetic ranking of different combinations of two or three flow modes in addition to the selfevident normal operation can be derived.

The low pressure short circuit regeneration (lpREGsc) and high pressure short circuit regeneration (hpREGsc) next to normal mode turns out to be a good trade-off between energyefficiency and complexity. This way the hydraulic pump energy consumption can be reduced to $68 \%$ theoretically compared to pure normal operation.

At this point it should be kept in mind that the results of the energetic potential analysis cannot be fully achieved in reality because of the time needed for mode switching, an effect not considered in the analysis. So the result of the analysis should be interpreted as a qualitative ranking rather than quantitative numbers. 


\subsection{Application tailored valve system}

The valve system used for the laboratory tests is depicted in Figure 6. For resolution purposes during slow lowering movements the meter out orifices in the subcircuits PATK and PATS as well as the short circuit flow from rod to piston side are mechanically pressure compensated. With this approach not only lowering but all movements are load compensated, as postulated in [11]. The concept described by Vucovic and Murrenhoff has been enhanced by pressure control valves in the inlet path to decouple the consumers from dynamic disturbances in the pump line. Apart from disturbance rejection the pressure control valves allow for fine pressure and force control and implement the directional switching function. Depending on the requirements on resolution and fine control and the characteristics of the used valves pressure and flow control could also be implemented electronically, like implied in Figure 3.

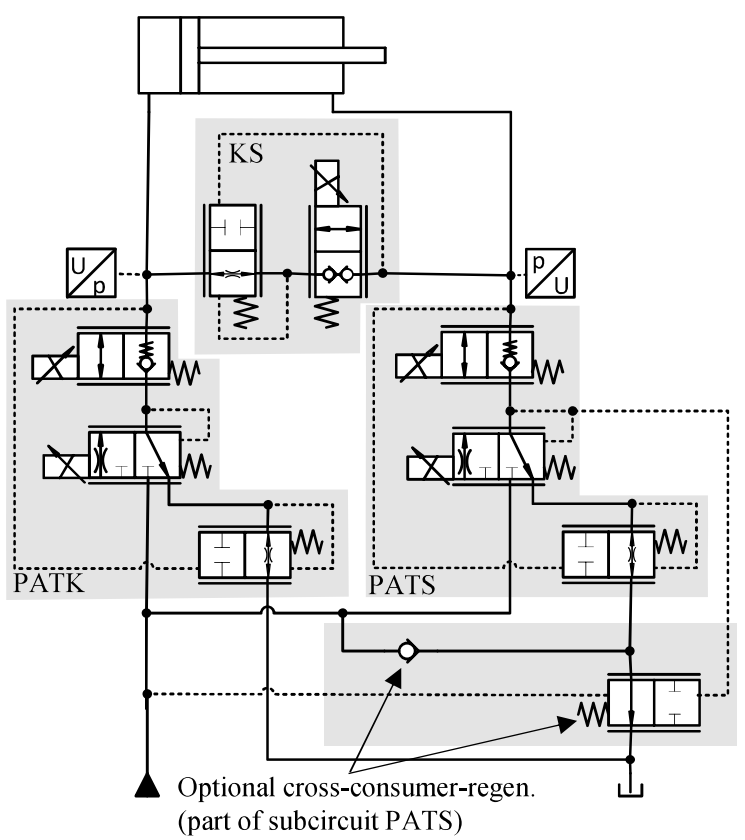

Figure 6: Test rig valve setup

The cross-consumer-regeneration subcircuit in the lower right corner of Figure 6 is an optional element that reroutes the outflow during a lowering movement (piston retraction with aiding load) back to the pump line to supply other consumers, in case the outflow pressure is higher than the pump pressure. In this case the switch valve in the lower right corner closes and the outflow oil passes the check valve to the pump line instead of flowing into the tank. In the control architecture the cross-consumer-regenerationelement belongs to the subcircuit PATS.

\section{SIMULATIVE EVALUATION}

\subsection{Simulation model and method}

The valve system has been modelled as a 1Dsystem with concentrated parameters, using the software-environment ESI SimulationX. For the simulations the circuit depicted in Figure 6 has been implemented for the boom, stick and bucket cylinder of an excavator arm, with the crossconsumer-regeneration-element only in the boom circuit since the boom is the only consumer with reasonable energetic recovery potential.

As throttling elements internally piloted seat valves are used and modelled as physical systems. The system is supplied by a displacement-controlled pump. Its control algorithm is designed as a feed-forward flowmatching-control combined with an LS-pressurecontroller, an adaption of the suggestions in [12]. The control code runs on a CoDeSys-based virtual ECU with a sampling time of $10 \mathrm{~ms}$. The ECU communicates with the simulation model via an OPC-server.

The cylinders move constant reduced masses of the example-excavators work implement (see section 4.1). For simulation of the work cycles of section 4.1 the velocity signals $v(t)=d x(t) / d t$ are fed to the controller while the forces $F(t)$ are acting on the cylinders at the same time. Individual PI position controllers for each axis act as a virtual operator who tries to follow the given position trajectories $x(t)$ as close as possible.

A conventional flow sharing LS-system of similar size serves as a reference for comparison purposes. It's LS pressure margin is $\Delta p_{L S}=$ 16 bar.

\subsection{Results}

Velocity Tracking. The simulation results for a pushed levelling cycle are shown in Figure 7: The reference trajectory $v_{\text {Des }}(t)$ is plotted with a black dotted line.

The conventional system's input velocity command is represented as a red dashed line while the actual velocity is red solid. The ideal behavior would be a perfect fit between reference, velocity command and actual velocity. 
However, especially during the lowering period of the boom between 4-8 and 12-15 s the actual velocity is much higher than the command. This is due to the load-dependent flow over the meterout-edge of the spool valve.

The behaviour of the proposed novel IM system is represented by the green lines, dashed for command and solid for actual velocity. As can be seen the actual velocity is much closer to the command due to the compensation of both resistive and aiding loads by the meter-out pressure compensation.

Even though an experienced operator is able to control a conventional system quite well, the machine itself - for the purpose of automation cannot. Especially in this case the IM system has clear advantages.

Mode Transition. Figure 8 shows the chamber pressures (red and green), the pump pressure $p_{0}$ (black dashed) and the volume flow from the pump to the corresponding consumer (black solid) for the IM systems on boom and stick during the pushed grading cycle from Figure 7. Furthermore, the plot is divided into several time sections, in which the IM circuits run in different flow modes (grey lines and headings).

An example for a mode shift during an ongoing movement can be seen at around $12 \mathrm{~s}$ :
From 8,5 to $12 \mathrm{~s}$ the boom lifts (see Figure 7 top) and thus raises the pump pressure $p_{0}$ to a high level of around 100 bar (Figure 8). The stick cylinder moves against a much lower load, causing the rod pressure to be much higher than the piston pressure (Figure 8 bottom). In this condition the stick operates in high pressure regeneration hpREGscK + . Once the boom stops extending at $12 \mathrm{~s}$ the pump pressure drops and so do the stick chamber pressures. The stick shifts back into normal operation NM+ smoothly, as can be seen at the continuous raise of its pump flow (black solid) between $12-12,5 \mathrm{~s}$ in Figure $\mathbf{8}$ bottom. The shifting event has only little impact on the cylinder velocity (green solid in Figure 7 bottom).

Energy efficiency. In the simulated pushed levelling cycle the novel IM system has saved $36 \%$ hydraulic pump output power compared to the conventional flow sharing system. This is mainly achieved by the reduction of pump flow by means of regenerative flow modes. In the whole operation cycle defined in section 4.1 approximately $30 \%$ have been saved. An exact number cannot be given because the measured digging cycle, which has been used for the potential analysis as a whole, is very long and
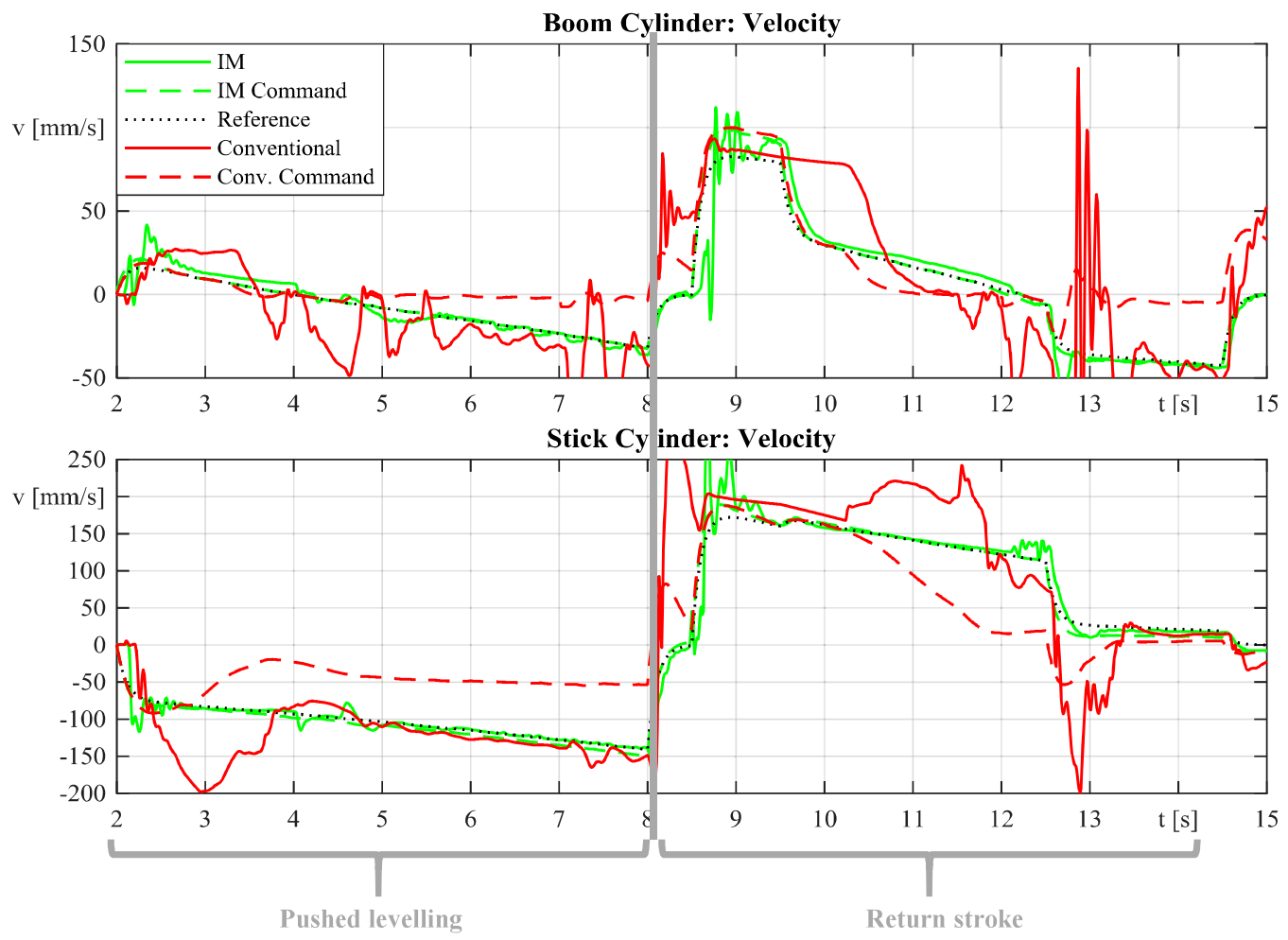

Figure 7: Velocity tracking during pushed levelling for conventional flow sharing and novel IM system 
therefore has not been completely simulated with the valve system model in section 5.1.

\section{SUMMARY AND OUTLOOK}

Smooth mode switching plays a major key role in exploiting the benefits of independent metering, especially in mobile applications. In this project, the approach of continuously variable modes in [7] has been adapted to a valve layout with short circuit path, thus enabling smooth switching with only three proportional flow paths. A flexible software structure has been derived from a general valve structure. The software is able to control a very broad variety of IM valve circuits. According to the results of an energetic analysis for typical work cycles of a medium sized excavator the three modes normal operation and high and low pressure short circuit regeneration have been identified as a good trade-off between cost and engineering effort on the one hand side and energy efficiency on the other.

On the basis of these results and functional requirements a valve layout for excavators has been developed and set up on a test rig. While the test rig is still under commissioning, 1Dsimulations with concentrated parameters already show promising results: Smooth mode switching with very low impact on the velocity and up to $36 \%$ reduction of hydraulic pump output energy. Measurements in terms of mode transition, velocity tracking, force control and energy efficiency will be carried out soon within 2020 .

\section{NOMENCLATURE}

$\begin{array}{ccl}0 & & \text { Supply line } \\ A & \mathrm{~mm}^{2} & \text { Piston Area } \\ \alpha & & \text { CVM shift variable } \\ \text { CVM } & & \text { Continuously Variable Mode } \\ \text { Des } & & \text { Desired } \\ F & N & \text { Force } \\ I M & & \text { Independent Metering } \\ K & & \text { Piston side } \\ K & l /(\min \cdot \sqrt{b a r}) & \text { Flow Coefficent } \\ L S & & \text { Load Sensing } \\ m e a s & & \text { Measured } \\ O C & & \text { Open Centre } \\ P & & \text { Pump } \\ p & b a r & \text { Pressure } \\ P C & & \text { Pressure Control } \\ Q & l / m i n & \text { Volume flow } \\ \text { Req } & & \text { Required } \\ S & & \text { Rod side } \\ T & & \text { Tank } \\ t & s & \text { Time } \\ T C P & & \text { Tool Centre Point } \\ v & \mathrm{~mm} / \mathrm{s} & \text { Velocity } \\ x & \mathrm{~mm} & \text { Position } \\ & & \end{array}$

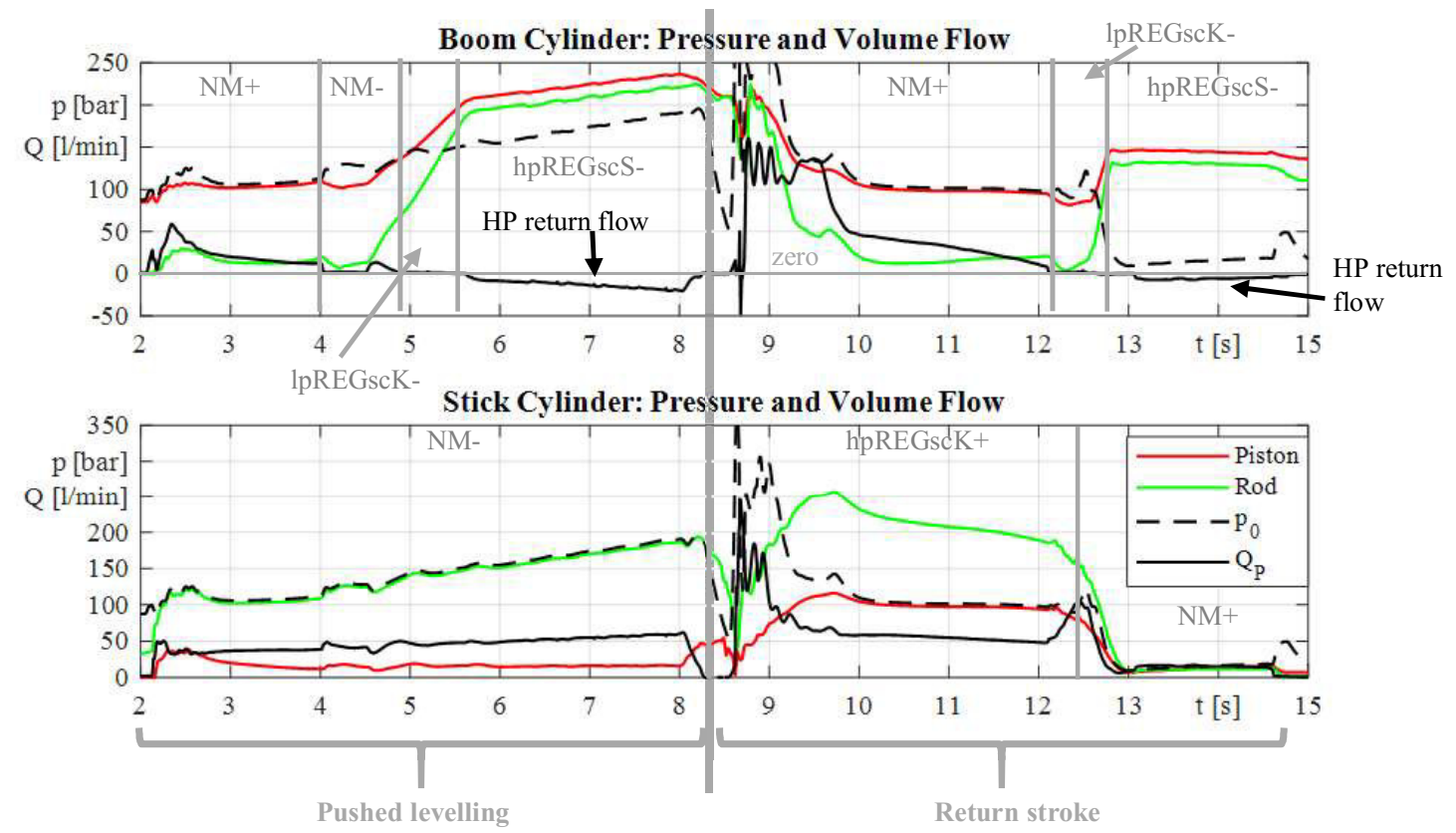

Figure 8: Chamber pressures and flow modes of IM system during pushed levelling 


\section{REFERENCES}

1. Finzel R (2010) Elektrohydraulische Steuerungssysteme für mobile Arbeitsmaschinen. Dissertation, TU Dresden

2. Sitte A, Weber J (2013) Structural design of independent metering control systems. In: Proceedings of the 13th Scandinavian International Conference on Fluid Power. Linköping (S)

3. Lee J-C, Jin K-C, Kwon Y-M, et al (2016) Development of the Independent Metering Valve Control System and Analysis of its Performance for an Excavator. In: Proceedings of the BATH/ASME 2016 Symposium on Fluid Power and Motion Control. Bath, UK

4. EATON CMA200 Advanced IndependentMetering Mobile Valve. Product data sheet by EATON Company. https://www.eaton.com/ecm/groups/public/@pu b/@eaton/@hyd/documents/content/pct_14519 17.pdf. Accessed 11 Feb 2019

5. Danfoss (2016) Proportional Valve PVX (product data sheet). https://assets.danfoss.com/documents/DOC152 986483080/DOC152986483080.pdf

6. Wessel Hydraulik GmbH (2013) Proportional valve with Autonomous Spools (Product data sheet).

http://www.hydpac.com/lib/common/download. php? fpath $=/$ data/bbs/solution/237.pdf\&fname $=I$ nformationsbrochure_PAS_20_28EN_29.pdf

7. Shenouda A (2006) Quasi-Static Hydraulic Control Systems and Energy Savings Potential Using Independent Metering Four-Valve Assembly Configuration. Dissertation, Woodruff School of Mechanical Engineering, Georgia Institute of Technology

8. Husco (2007) INCOVA ${ }^{\circledR}$ Hydraulic Control System for Excavators (Product data sheet by Husco).

http://hydrodream.ru/upload/iblock/e56/e5619a 3a7f4flaed0b1be538c1b0e743.pdf. Accessed 3 May 2019

9. Kolks G, Weber J (2016) Modiciency - Efficient industrial hydraulic drives through independent metering using optimal operating modes. In: Proceedings of the 10. International Fluid Power Conference. Dresden

10. Ding R, Xu B, Zhang J, Cheng M (2016) Bumpless mode switch of independent metering fluid power system for mobile machinery. Autom Constr 68:52-64
11. Vukovic M, Murrenhoff H (2014) Single Edge Meter Out Control for Mobile Machinery. In: Proceedings of the ASME/BATH 2014 Symposium on Fluid Power \& Motion Control FPMC2014. Bath (UK)

12. Djurovic M (2007) Energiesparende Antriebssysteme für die Arbeitshydraulik mobiler Arbeitsmaschinen „Elektrohydraulisches Flow Matching“. Dissertation, TU Dresden 